\title{
Real-World Clinical Practice Use of 8-Week Glecaprevir/Pibrentasvir in Treatment-Naïve Patients with Compensated Cirrhosis
}

\author{
Pietro Lampertico (D) - Stefan Mauss · Marcello Persico · \\ Stephen T. Barclay · Steven Marx · Kristina Lohmann • \\ Mark Bondin - ZhenZhen Zhang - Fiona Marra - Pamela S. Belperio • \\ Heiner Wedemeyer $\cdot$ Steven Flamm
}

Received: June 12, 2020 / Published online: August 4, 2020

(C) The Author(s) 2020

\section{ABSTRACT}

Introduction: More than 70 million people are estimated to be infected with hepatitis $C$ virus globally. Glecaprevir/pibrentasvir is a widely

Digital Features To view digital features for this article go to https://doi.org/10.6084/m9.figshare.12652802.

\section{P. Lampertico $(\varangle)$}

Division of Gastroenterology and Hepatolgy, CRC

"A.M. and A.Migliavacca" Center for Liver Disease, Foundation IRCCS Ca' Granda Ospedale Maggiore Policlinico, Milan, Italy

e-mail: pietro.lampertico@unimi.it

\section{P. Lampertico}

Department of Pathophysiology and

Transplantation, University of Milan, Milan, Italy

S. Mauss

Center for HIV and Hepatogastroenterology,

Düsseldorf, Germany

M. Persico

Internal Medicine and Hepatology Unit, University of Salerno, Salerno, Italy

\section{S. T. Barclay}

Department of Life Sciences, Glasgow Caledonian

University, Glasgow, UK

\section{S. T. Barclay}

Gastroenterology, Glasgow Royal Infirmary,

Glasgow, UK

S. Marx $\cdot$ M. Bondin $\cdot$ Z. Zhang

AbbVie Inc., North Chicago, IL, USA used treatment and has recently been approved for an 8-week regimen for treatment-naïve patients with compensated cirrhosis in Europe and the USA, who would previously have received glecaprevir/pibrentasvir for 12 weeks. This label update was based on the EXPEDITION-8 study, which included 343 treatment-naïve patients with compensated cirrhosis. However, there is currently a lack of similarly large-scale

\section{K. Lohmann}

AbbVie Deutschland GmBH Co. KG, Wiesbaden, Germany

\section{F. Marra}

University of Liverpool Hepatology Drug

Interactions Group, Liverpool, UK

\section{P. S. Belperio}

Department of Veterans Affairs, VA Palo Alto

Healthcare System, Palo Alto, CA, USA

\section{H. Wedemeyer}

Department of Gastroenterology and Hepatology, Essen University Hospital, Essen, Germany

\section{H. Wedemeyer}

Leberstiftungs-GmbH Deutschland, Hannover, Germany

\section{H. Wedemeyer}

Department of Gastroenterology, Hepatology and Endocrinology, Hannover Medical School, Hannover, Germany

S. Flamm

Northwestern University Feinberg School of Medicine, Chicago, IL, USA 
real-world studies of the 8-week glecaprevir/pibrentasvir regimen in this population.

Methods: This summary of seven separate smaller real-world studies aims to validate the results seen in EXPEDITION-8 and provide an up-to-date real-world reference for clinicians making treatment decisions for patients with compensated cirrhosis (Child-Pugh A) who may benefit from a shorter-duration therapy with glecaprevir/pibrentasvir. The newly emerging real-world effectiveness data on treatment-naïve patients with compensated cirrhosis treated with 8 weeks of glecaprevir/pibrentasvir help to understand where further research is needed to support patients with hepatitis $C$ virus.

Results: Across all seven studies, glecaprevir/ pibrentasvir showed high effectiveness with an average sustained virologic response rate of $98.1 \%$, similar to that found in a clinical trial setting (99.7\%). Only one patient $(0.5 \%)$ experienced virologic failure and treatment was well tolerated.

Conclusion: Expanding the number of patients eligible for the shortened treatment duration will potentially increase treatment initiation and completion, particularly in underserved populations, contributing to the elimination of hepatitis $C$ virus.

Keywords: Fibrosis; Hepatitis C; Infectious disease; Review; Therapeutics

\section{Key Summary Points}

Why carry out this study?

Short-duration, pangenotypic directacting antivirals (DAA), such as glecaprevir/pibrentasvir $(\mathrm{G} / \mathrm{P})$, are increasingly important therapies, which can support countries' paths to meet the World Health Organization's target to eliminate hepatitis $\mathrm{C}$ virus (HCV).
Following a recent label (SmPC and USPI) update for G/P based on the EXPEDITION8 study, there is a lack of large-scale realworld studies of similar magnitude (343 patients) for 8-week G/P therapy in treatment-naïve (TN) patients with compensated cirrhosis (CC). This review of smaller real-world studies will provide an up-to-date reference for clinicians making treatment decisions for patients with CC that may benefit from a shorterduration therapy with G/P.

This review summarizes data from seven independent real-world studies to examine the effectiveness and safety of $\mathrm{G} / \mathrm{P}$ treatment in $\mathrm{HCV}$-infected TN patients with CC in clinical practice.

\section{What was learned from the study?}

G/P therapy for 8 weeks showed high effectiveness, with numerically high sustained virologic response rates at posttreatment week 12 (an average of $98.1 \%$ across cohorts), and was well tolerated.

This review can reassure providers that the results obtained across smaller real-world studies are collectively similar to that of EXPEDITION-8, where 343 patients were enrolled. This shorter treatment for TN patients with CC may also be beneficial in hard-to-reach populations.

\section{INTRODUCTION}

Effective pangenotypic hepatitis $\mathrm{C}$ virus (HCV) treatment is available through all-oral directacting antiviral (DAA) therapies [1]. However, despite the availability of these treatments, prevalence of HCV remains high, often due to a lack of diagnosis and treatment [2]. In 2016, it was reported that approximately $13 \%$ of patients aware of their positive HCV status were being treated [3].

If left untreated, chronic HCV infection can lead to complications such as hepatic 
decompensation and hepatocellular carcinoma, ultimately leading to death [1]. Untreated HCV infection may also lead to additional routes of transmission, particularly in high-risk groups [4]. The World Health Organization (WHO) has set a target to eliminate viral hepatitis as a major public health threat by 2030 by reducing new hepatitis B and C infections by $90 \%$ and hepatitis mortality by $65 \%$ [5]. However, many countries are not on track to meet the elimination targets by 2030 [3].

Glecaprevir/pibrentasvir $(\mathrm{G} / \mathrm{P})$ is an interferon-free, ribavirin-free, fixed-dose DAA drug combination approved for the treatment of chronic HCV genotype (GT) 1-6 [6, 7], with studies showing high rates of sustained virologic response (SVR) and well-tolerated safety profiles [8]. Recently, the EXPEDITION-8 trial demonstrated the efficacy and tolerability of G/P in 343 patients without any previous $\mathrm{HCV}$ treatment (treatment naïve [TN]) with compensated cirrhosis (CC) receiving treatment for 8 weeks [8]. These data supported an update of the $\mathrm{G} / \mathrm{P}$ treatment label to include TN patients with CC for the 8-week regimen for GT1-6 in Europe [6], and the USA [7], whereas previously only patients without cirrhosis (either treatment-experienced patients with GT1, 2, 4-6 and TN patients with GT1-6) were eligible for this treatment duration. However, following this recent label update, there remains a lack of large-scale real-world data of a similar scale to the EXPEDITION-8 trial for 8-week G/P therapy in TN patients with CC. In lieu of a large prospective real-world cohort, this review summarizes data from seven smaller real-world studies to provide an up-to-date reference for clinicians making treatment decisions for patients with CC who may benefit from a shorter treatment duration.

Increasing the number of patients eligible for the shorter 8-week treatment with G/P has the potential to reach newly diagnosed $\mathrm{TN}$ patients by addressing barriers in the care cascade. It may also potentially offer additional advantages such as improved patient uptake [9] due to reduced treatment duration and monitoring, as well as improved adherence in patients who use drugs [10]. This review of data from seven separate cohort studies aims to summarize newly emerging data on TN patients with CC (Child-Pugh A) and HCV treated in real-world clinical practice with 8 weeks of G/P treatment and to further understand where additional real-world data are needed for newly diagnosed patients with HCV across multiple populations.

\section{METHODS}

\section{Study Selection}

A literature synthesis of PubMed, American Association for the Study of Liver Disease Congress abstracts, and National AIDS Treatment Advocacy Project abstracts was conducted from August 2018 to April 2020 to identify publications reporting real-world evidence of 8 -week G/P in TN patients with CC. All seven studies were retrospective, of which three were published or peer-reviewed published manuscripts and four were presented at major liver meetings. As this was a summary of published real-world studies, no ethic committee review was required. All studies included in this summary were approved by local institutional review committees, each patient also gave written informed consent before enrollment, and the protocol was conducted in accordance with the Declaration of Helsinki, with the exception of the Veterans Association study which did not require patient informed consent because the study was observational, the Scottish HCV study where data were obtained from the Scottish Hepatitis C Database for which opt-out consent is in place, and the TRIO study where TRIO Health Analytics were provided with deidentified Health Insurance Portability and Accountability Act-compliant patient information approved by the Western Institutional Review Board.

\section{Patient Populations}

We present a review of individual real-world effectiveness data from seven separate studies of TN patients with CC who were given G/P for 8 weeks. CC (Child-Pugh A) was defined as cirrhosis with no prior history of liver 
decompensation, consistent with label indicated populations [6, 7]. Decompensated cirrhosis was defined as current or past evidence of Child-Pugh B or C classification or clinical history of liver decompensation including ascites, bleeding esophageal varices, or hepatic encephalopathy, with the exception of the German hepatitis C-registry (DHC-R) which defined decompensated cirrhosis as at least one of the following: Child-Pugh B or C classification; ascites; encephalopathy; esophageal-varices grade $>1$, red spots, bleeding; or model for end-stage liver disease score $>15$.

\section{Effectiveness Analyses}

Effectiveness was measured using the SVR at post-treatment week 12 (SVR12). For the global post-marketing observation studies (PMOS), the core population with sufficient follow-up (CPSFU), which included patients who completed the label-recommended regimen and the availability of HCV ribonucleic acid (RNA) data after post-treatment day 70 , virologic failure or discontinuation due to an adverse event with $\mathrm{HCV}$ RNA $<50 \mathrm{IU} / \mathrm{mL}$ at the last measurement was used for SVR12 analysis [11]. The Scottish HCV [12] and Italian MISTRAL [13] studies used the intention-to-treat (ITT) population, which included all patients. The DHC-R [14] used the modified ITT (mITT) population, which excluded patients lost to follow-up. The Italian NAVIGATORE [15], US TRIO Health Analytics [16], and US Department of Veterans Affairs (VA) [17] studies used the per-protocol (PP) population, which included patients who completed treatment. Safety profile, in terms of deaths and adverse events (AEs) where available, were assessed in TN patients with CC treated with $\mathrm{G} / \mathrm{P}$ for 8 weeks.

\section{RESULTS}

Data from a total of seven studies are presented: global PMOS [11], DHC-R [14], Scottish HCV [12], Italian MISTRAL [13], Italian NAVIGATORE [15], US TRIO Health Analytics [16], and US VA [17], with additional 8-week TN CC-specific data provided for inclusion in this review. Baseline patient demographics and clinical characteristics, including GT and fibrosis stage, are presented in Table 1.

\section{Efficacy}

SVR12 rates were high (>95.5\%) across the majority of real-world studies included (Fig. 1).

\section{Safety}

Of the seven studies, five (PMOS, Scottish HCV, MISTRAL, NAVIGATORE, and VA) had available safety data for TN patients with CC treated with G/P for 8 weeks. Across the 135 patients included in these cohorts, a total of two deaths were reported, 1/24 (4.2\%) in the Scottish HCV study and $1 / 85(1.2 \%)$ in the VA study $[11-13,15,17]$. Both deaths occurred post treatment and were not deemed DAA related.

There were substantial differences in the methodologies of safety reporting across the studies. However, the following safety profiles were available at the time of publication. In the PMOS study, 2/12 (16.7\%) patients experienced an $\mathrm{AE}$ and no patients experienced a serious $\mathrm{AE}$ (SAE). In the Scottish HCV study, there were no significant AEs attributed to the study drug, and one death post treatment due to an illicit drug overdose. In the MISTRAL and NAVIGATORE studies there were no AEs reported. In the DHC-R study, the safety profile was only available for $16 / 20$ patients, with no patient experiencing an SAE. The TRIO study was an effectiveness-only analysis and therefore no safety data were available. In the VA study there was one death post treatment but before SVR testing which was not deemed treatment related.

\section{DISCUSSION}

In general, it seems possible that the effectiveness of HCV treatments in real-world clinical practice may be lower than that reported in clinical trials, potentially due to a more diverse population [18]. It is therefore important to examine effectiveness of $\mathrm{HCV}$ treatments in real-world populations given the consequences 


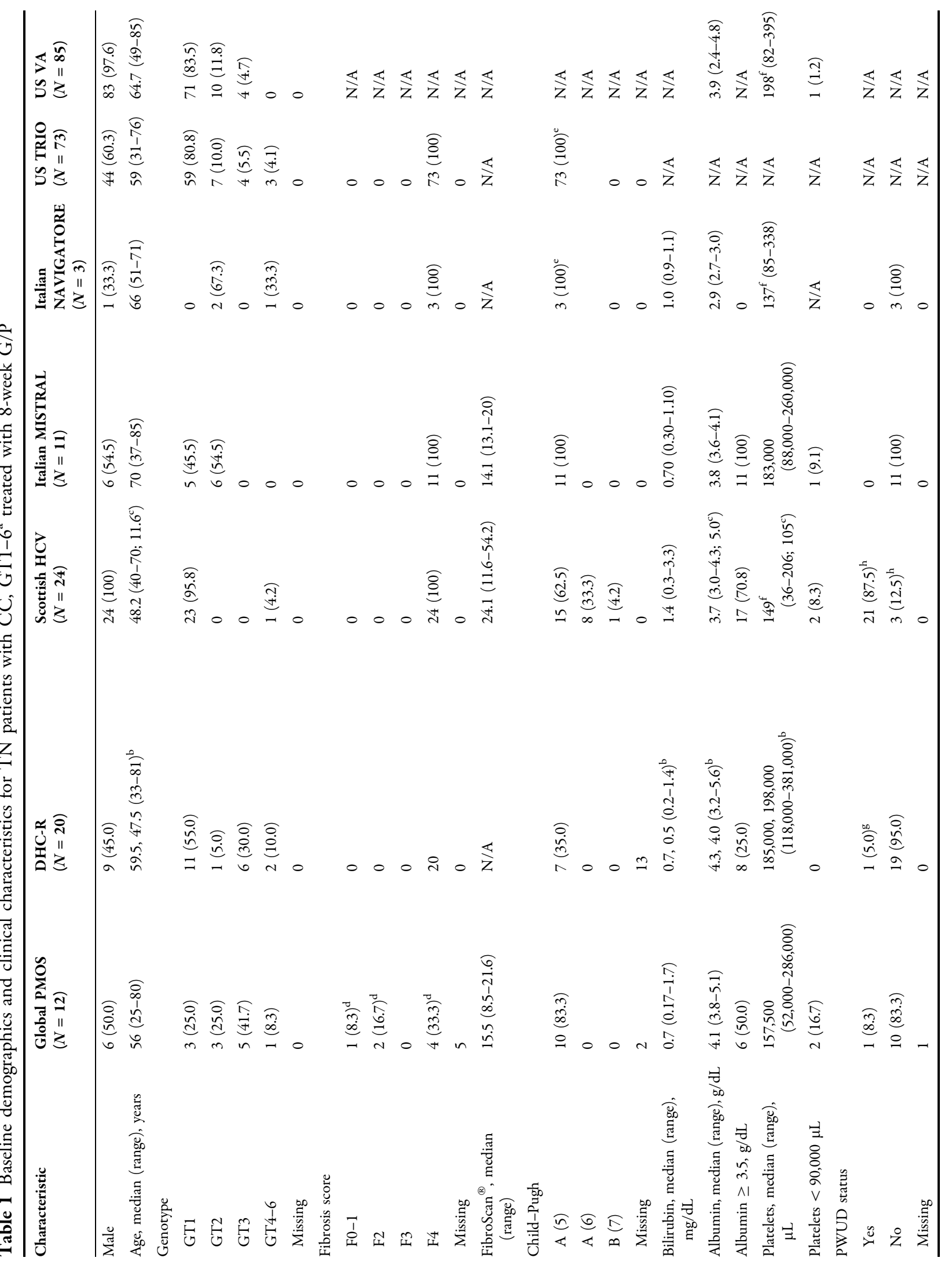




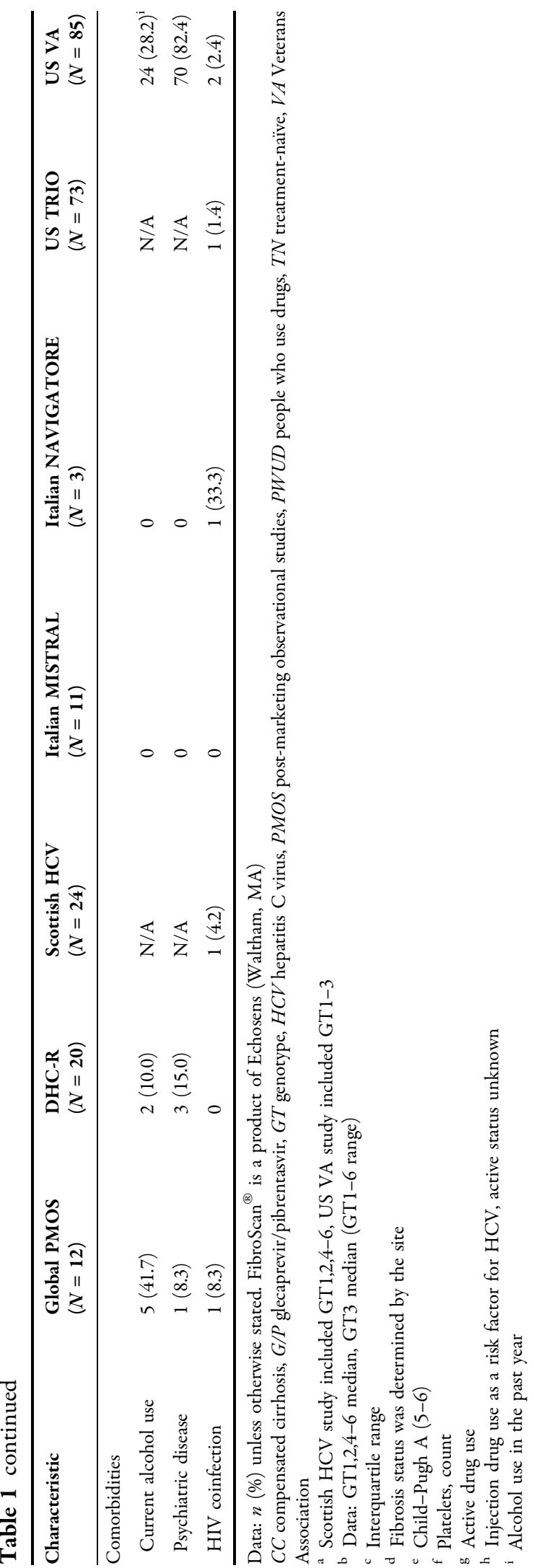

of treatment failure. Early real-world experience from these seven individual studies demonstrates the effectiveness of the 8-week G/P regimen in TN patients with CC with $212 / 216$ (98.1\%) patients achieving SVR and only 1/216 $(0.5 \%)$ experiencing virologic failure. Further real-world studies are needed to support these results. Across most studies, the majority of patients were GT1, except the PMOS, MISTRAL, and NAVIGATORE studies where the majority of patients were GT3 (41.7\%), GT2 (54.5\%), and GT2 (67.3\%), respectively [11-15]. Additional effectiveness and safety data, including migrants, prisoners, men who have sex with men, patients with mental health diagnoses, patients with comorbidities, and more descriptive drug use should be considered in future studies, as well as the under-representation of GT3, GT5, and GT6. However, patients with other GTs have shown similar real-world results as seen in clinical trials, providing reassurance to the applicability of a shortened treatment duration. Further research is also required on the potential for drug-drug interaction in $\mathrm{TN}$ patients with $\mathrm{CC}$ receiving 8-week G/P treatment, and possibly further shortening of $\mathrm{HCV}$ treatment given the high effectiveness shown. In addition, future studies are needed to demonstrate the value of a shorter treatment duration within test and treat programs and point-of-care approaches, where diagnosis and treatment are given in a single visit [19].

The heterogenous data summarized in this review are limited to the data available at the time of publication of the seven separate cohorts. Limitations arising from summarizing data captured from seven separate cohorts include the lack of uniform data collection and the limited utility of pooling data due to the different methodologies and study populations. Another limitation of this review is the use of mITT and CPSFU populations in some studies, which do not include missing SVR data for patients lost to follow-up and therefore only present data for patients who completed followup. This could potentially lead to underreported relapses and could potentially affect the conclusions drawn.

The majority of the real-world SVR12 rates presented here were similar to the results of the 


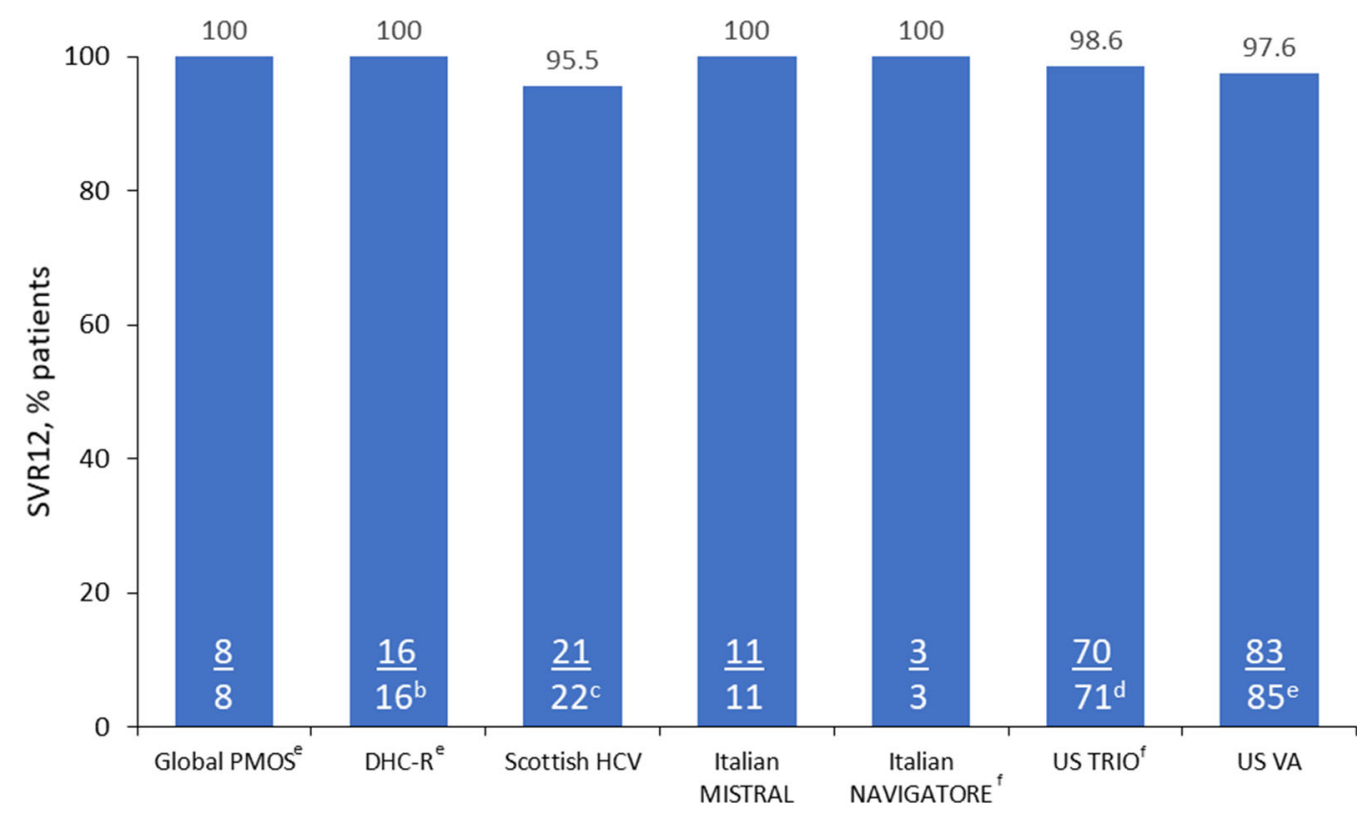

Fig. 1 SVR12 rates in TN patients with CC, GT1-6 ${ }^{\mathrm{a}}$

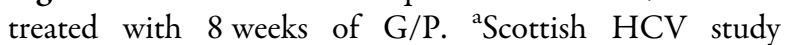
included GT1,2,4-6, US VA study included GT1-3. ${ }^{\mathrm{b}}$ Four patients were lost to follow-up in the ITT population. ${ }^{\mathrm{C}}$ One confirmed reinfection with subsequent spontaneous clearance, no virologic failure. ${ }^{\mathrm{d}}$ One virologic failure. ${ }^{\mathrm{e}}$ One patient died after completing treatment but before SVR12 testing, one patient was lost to follow-up.

EXPEDITION 8 registration study (PP: 99.7\% [334/335]) [8], which led to a G/P label update to include TN patients with $\mathrm{CC}$ for the 8-week regimen for GT1-6 in Europe [6] and the USA [7]. This similar efficacy is an important finding from this collection and review of smaller cohorts, which can be used in lieu of a largescale prospective real-world study of a similar cohort size as in EXPEDITION-8. Increasing the number of patients with $\mathrm{HCV}$ who are eligible for shorter treatment duration has the potential to improve treatment initiation and completion, as well as reduce healthcare costs. This more inclusive indication for the 8-week treatment duration is of particular importance for TN patients with CC, in whom only a 12 -week duration was previously possible, providing a new option for a shorter 8-week course of therapy to treat their HCV infection. Furthermore, the TN HCV-infected population is growing [20], with the majority of treatment-

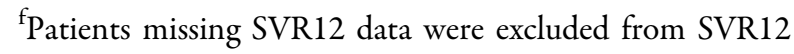
analysis. CC compensated cirrhosis, DHC-R German Hepatitis C-Registry, $G / P$ glecaprevir/pibrentasvir, $G T$ genotype, $H C V$ hepatitis $\mathrm{C}$ virus, ITT intention-to-treat, PMOS post-marketing observational studies, SVR 12 sustained virologic response at week $12, T N$ treatment-naïve, $V A$ Veterans Association

experienced patients successfully re-treated with DAAs. Epidemiology estimates suggest that as many as $98 \%$ of TN patients with HCV would have no cirrhosis or CC and therefore could be treated with, and are amenable to, the 8-week regimen [21]. In the USA, TN patients without cirrhosis and with CC represent $98 \%$ of the HCV patient population [16]; therefore, the majority of patients are eligible for 8-week treatment. Although similar high SVR12 rates are observed for the 12-week $G / P$ regimen, patients may prefer a shorter duration because it is more convenient and requires less monitoring [9].

\section{CONCLUSION}

This summary of real-world data demonstrates that 8-week G/P is effective and well tolerated in TN patients with CC, supporting the results of EXPEDITION-8 and providing reassurance to 
clinicians treating patients with CC. Reducing treatment duration may help to address remaining gaps in the cascade of care, such as a lack of treatment initiation on behalf of either the patient or the physician and losing patients to follow-up. It may have greater application in underserved patients, such as prisoners and those with unstable lifestyles/addictive substance use whose engagement with healthcare can be challenging.

\section{ACKNOWLEDGEMENTS}

AbbVie sponsored the study; contributed to its design; and participated in the collection, analysis, and interpretation of the data and in the writing, reviewing, and approval of the publication. Glecaprevir was identified by AbbVie and Enanta.

Funding. The studies were sponsored by AbbVie, Gilead and MSD, with the exception of the US Department of Veterans Affairs study, which was conducted independently. AbbVie funded the journal's Rapid Service Fees.

Medical Writing, Editorial, and Other Assistance. Medical writing support was provided by Annie Massa, MBiolSci, and Heather Shawcross, PhD, of Fishawack Communications Ltd, funded by AbbVie.

Authorship. All named authors meet the International Committee of Medical Journal Editors (ICMJE) criteria for authorship for this article, take responsibility for the integrity of the work as a whole, and have given their approval for this version to be published. No honoraria or payments were made for authorship.

Authorship Contributions. All authors had access to relevant data, and participated in the writing, review, and approval of the final presentation.

Disclosures. Pietro Lampertico: Speaker bureau and/or advisory board: AbbVie, Alnylam, Arrowhead, Bristol-Myers Squibb,
Eiger BioPharmaceuticals, Gilead Sciences, GSK, Janssen, MSD, MYR Pharmaceuticals, Roche and Spring Bank. Stefan Mauss: Speaker and advisory board: AbbVie and MSD. Speaker: Gilead and Janssen. Marcello Persico: Consultant: Gilead, MSD, and AbbVie. Stephen T. Barclay: Received speaker and teaching fees and grant/ research support: AbbVie and Gilead. Advisory board: Gilead. Steven Marx, Kristina Lohmann, Mark Bodin and Zhenzhen Zhang are employees of AbbVie and may hold stock/share options. Fiona Marra: Received consulting or teaching fees: AbbVie, Gilead, MSD and ViiV. Pamela S. Belperio has nothing to disclose. Heiner Wedemeyer: Honoraria for consulting/ speaking: Abbott, AbbVie, Bristol-Myers Squibb, Gilead, Janssen, Merck/MSD, and Roche. Steven Flamm: Speaker bureau for, advises for, and received grants: AbbVie and Gilead. Advises: Merck.

Compliance with Ethics Guidelines. All studies included in this summary were approved by local institutional review committees, each patient also gave written informed consent before enrollment, and the protocol was conducted in accordance with the Declaration of Helsinki, with the exception of the Veterans Association study which did not require patient informed consent because the study was observational, the Scottish HCV study where data were obtained from the Scottish Hepatitis C Database for which opt-out consent is in place, and the TRIO study where TRIO Health Analytics were provided with deidentified Health Insurance Portability and Accountability Act-compliant patient information approved by the Western Institutional Review Board.

Data Availability. AbbVie is committed to responsible data sharing regarding the clinical trials we sponsor. This includes access to anonymized, individual and trial-level data (analysis data sets), as well as other information (e.g., protocols and Clinical Study Reports), as long as the trials are not part of an ongoing or planned regulatory submission. This includes requests for clinical trial data for unlicensed products and indications. This clinical trial data can be 
requested by any qualified researchers who engage in rigorous, independent scientific research, and will be provided following review and approval of a research proposal and Statistical Analysis Plan (SAP) and execution of a Data Sharing Agreement (DSA). Data requests can be submitted at any time and the data will be accessible for 12 months, with possible extensions considered. For more information on the process, or to submit a request, visit the following link: https://www.abbvie.com/ourscience/clinical-trials/clinical-trials-data-andinformation-sharing/data-and-information-shar ing-with-qualified-researchers.html. The datasets analysed during the current study are available from the following sources: Global PMOS: Lampertico P, Peck-Radosavljevic M, Bondin $\mathrm{M}$, et al. Addressing barriers to hepatitis $\mathrm{C}$ virus (HCV) elimination: real-world outcomes in historically underserved patients with chronic HCV infection treated with glecaprevir/ pibrentasvir. AASLD 2019 Poster 1583 (https:// www.natap.org/2019/AASLD/AASLD_10.htm). DHC-R: Wedemeyer H, Erren P, Naumann U, et al. Glecaprevir/pibrentasvir is effective and well tolerated in hepatitis $C$ patients with cirrhosis: real-world experience from the German Hepatitis C-Registry. AASLD 2019 Poster 1525 (https://www.natap.org/2019/AASLD/AASLD_

11.htm). Scottish HCV: Marra F, Boyle A, McDonald $\mathrm{N}$, et al. 8 weeks of glecaprevir/pibrentasvir is effective and well tolerated in nonGT3 HCV patients with cirrhosis. AASLD 2019 Poster 1588 (https://www.natap.org/2019/ AASLD/AASLD_85.htm). Italian MISTRAL: Persico M, Aglitti A, Milella M, et al. Real-life glecaprevir/pibrentasvir in a large cohort of patients with hepatitis $C$ virus infection: the MISTRAL study. Liver Int. 2019;39:1852-9 (https://pubmed.ncbi.nlm.nih.gov/31175707/). Italian NAVIGATORE: D'Ambrosio R, Pasulo L, Puoti $\mathrm{M}$, et al. Real-world effectiveness and safety of glecaprevir/pibrentasvir in 723 patients with chronic hepatitis C. J Hepatol. 2019;70:379-87 (https://pubmed.ncbi.nlm.nih. gov/30472321/). US TRIO: Flamm S, Kort J, Marx S, et al. Effectiveness of 8-week glecaprevir/pibrentasvir for treatment naive, compensated cirrhotic patients with chronic hepatitis C infection. Adv Ther. 2020;37:2267-74 (https:// pubmed.ncbi.nlm.nih.gov/32279176/). US VA: Belperio P, Shahoumian T, Loomis T, Mole L, Backus L. Real-world effectiveness of glecaprevir/pibrentasvir in 1,941 patients with hepatitis C genotypes 1 through 4. Hepatology. 2018;68:417A. Abstract 703 (https://www. natap.org/2018/AASLD/AASLD_23.htm).

Open Access. This article is licensed under a Creative Commons Attribution-NonCommercial 4.0 International License, which permits any non-commercial use, sharing, adaptation, distribution and reproduction in any medium or format, as long as you give appropriate credit to the original author(s) and the source, provide a link to the Creative Commons licence, and indicate if changes were made. The images or other third party material in this article are included in the article's Creative Commons licence, unless indicated otherwise in a credit line to the material. If material is not included in the article's Creative Commons licence and your intended use is not permitted by statutory regulation or exceeds the permitted use, you will need to obtain permission directly from the copyright holder. To view a copy of this licence, visit http:// creativecommons.org/licenses/by-nc/4.0/.

\section{REFERENCES}

1. World Health Organization (WHO). Hepatitis C key facts. 2019. https://www.who.int/en/news-room/ fact-sheets/detail/hepatitis-c. Accessed Mar 2020.

2. Lombardi A, Mondelli M, ESCMID Study Group for Viral Hepatitis. Hepatitis C: is eradication possible? Liver Int. 2018;39(3):416-26.

3. World Health Organization (WHO). Progress report on access to hepatitis C treatment. 2018. https:// apps.who.int/iris/bitstream/handle/10665/260445/ WHO-CDS-HIV-18.4-eng.pdf; jsessionid=AC7402A DBBC3BA2404E5DF4768BE7F9B?sequence $=1$. Accessed Mar 2020.

4. Midgard H, Weir A, Palmateer N, et al. HCV epidemiology in high-risk groups and the risk of reinfection. J Hepatol. 2016;65(1 Suppl):S33-S45.

5. World Health Organization (WHO). Global health sector strategy on viral hepatitis, 2016-2021. 2016. 
https://apps.who.int/iris/bitstream/handle/10665/ 246177/WHO-HIV-2016.06-eng.pdf;jsessionid=B789 DC827B02AF7A18F792AB221CF0D5? sequence $=1$. Accessed Mar 2020.

6. Maviret EU. Maviret. 2020. https://www.ema. europa.eu/en/documents/product-information/ maviret-epar-product-information_en.pdf. Accessed June 2020.

7. Mavyret US. Mavyret US. 2019. https://www. rxabbvie.com/pdf/mavyret_pi.pdf. Accessed Mar 2020.

8. Brown RS Jr, Buti M, Rodrigues L, et al. Glecaprevir/ pibrentasvir for 8 weeks in treatment-naive patients with chronic HCV genotypes 1-6 and compensated cirrhosis: the EXPEDITION-8 trial. J Hepatol. 2019;72(3):441-9.

9. Welzel TM, Yang M, Sajeev G, et al. Assessing patient preferences for treatment decisions for new direct acting antiviral (DAA) therapies for chronic hepatitis C virus infections. Adv Ther. 2019;36(9): 2475-86.

10. Akiyama MJ, Norton BL, Arnsten JH, Agyemang L, Heo M, Litwin AH. Intensive models of hepatitis C care for people who inject drugs receiving opioid agonist therapy: a randomized controlled trial. Ann Intern Med. 2019;170(9):594-603.

11. Lampertico P, Peck-Radosavljevic M, Bondin M, et al. Addressing barriers to hepatitis $\mathrm{C}$ virus (HCV) elimination: real-world outcomes in historically underserved patients with chronic HCV infection treated with glecaprevir/pibrentasvir. AASLD 2019 Poster 1583.

12. Marra F, Boyle A, McDonald N, et al. 8 weeks of glecaprevir/pibrentasvir is effective and well tolerated in non-GT3 hcv patients with cirrhosis. AASLD 2019 Poster 1588.

13. Persico M, Aglitti A, Milella M, et al. Real-life glecaprevir/pibrentasvir in a large cohort of patients with hepatitis $C$ virus infection: the MISTRAL study. Liver Int. 2019;39(10):1852-9.

14. Wedemeyer H, Erren P, Naumann U, et al. Glecaprevir/pibrentasvir is effective and well tolerated in hepatitis $C$ patients with cirrhosis: real-world experience from the German Hepatitis C-Registry. AASLD 2019 Poster 1525.

15. D'Ambrosio R, Pasulo L, Puoti M, et al. Real-world effectiveness and safety of glecaprevir/pibrentasvir in 723 patients with chronic hepatitis C. J Hepatol. 2019;70(3):379-87.

16. Flamm S, Kort J, Marx S, et al. Effectiveness of 8-week glecaprevir/pibrentasvir for treatment naive, compensated cirrhotic patients with chronic hepatitis C infection. Adv Ther. 2020;37:2267-74.

17. Belperio P, Shahoumian T, Loomis $\mathrm{T}$, Mole $\mathrm{L}$, Backus L. Real-world effectiveness of glecaprevir/ pibrentasvir in 1,941 patients with hepatitis C genotypes 1 through 4. Hepatology. 2018;68(S1): 417A (Abstract 703).

18. Zoulim F, Liang TJ, Gerbes AL, et al. Hepatitis C virus treatment in the real world: optimising treatment and access to therapies. Gut. 2015;64(11): 1824-33.

19. Applegate TL, Fajardo E, Sacks JA. Hepatitis C virus diagnosis and the Holy Grail. Infect Dis Clin N Am. 2018;32(2):425-45.

20. Chirikov VV, Marx SE, Manthena SR, Strezewski JP, Saab S. Development of a comprehensive dataset of hepatitis $C$ patients and examination of disease epidemiology in the United States, 2013-2016. Adv Ther. 2018;35(7):1087-102.

21. Data on file. AbbVie Inc. Ipsos Healthcare HCV USA Therapy Monitor (July 16, 2018-March 2019, all data collected online) (CIpsos 2019, all rights reserved. 